EPJ Web of Conferences 60, 20028 (2013)

DOI: $10.1051 /$ epjconf $/ 20136020028$

(C) Owned by the authors, published by EDP Sciences, 2013

\title{
Search for the Standard Model Higgs boson produced in association with top quarks and decaying to $b \bar{b}$ at $\sqrt{s}=7 \mathrm{TeV}$
}

\author{
Javier Montejo Berlingen ${ }^{1 \mathrm{a}}$, on behalf of the ATLAS Collaboration \\ ${ }^{1}$ Institut de Física d'Altes Energies, Universitat Autònoma de Barcelona
}

\begin{abstract}
A search for a Higgs boson produced in association with a pair of top quarks $(t \bar{t} H)$ and decaying into a pair of bottom quarks $(H \rightarrow b \bar{b})$ is presented. The analysis uses $4.7 \mathrm{fb}^{-1}$ of $p p$ collision data at $\sqrt{s}=7 \mathrm{TeV}$ collected in 2011 with the ATLAS detector at the CERN Large Hadron Collider. The search is focused on the semileptonic decay mode of the $t \bar{t}$ system which, together with the $H \rightarrow b \bar{b}$ decay, typically results in a final state signature characterised by one high transverse momentum isolated electron or muon, high transverse missing momentum and six jets, of which four jets originate from $b$ quarks. Events are classified in nine different topologies depending on their jet and $b$-tagged jet multiplicities in order to improve the sensitivity of the search. No significant excess of events above the background expectation is observed and $95 \%$ confidencelevel upper limits on the production cross section times branching ratio, $\sigma(t \bar{t} H) \times B R(H \rightarrow b \bar{b})$, are derived for a Higgs boson with a mass between 110 and $140 \mathrm{GeV}$. For a Higgs boson with a mass of $125 \mathrm{GeV}$, an observed (expected) 95\% confidence-level upper limit of 13.1 (10.5) times the Standard Model cross section is obtained.
\end{abstract}

\section{Introduction}

The observation of a Higgs-like particle was reported by the ATLAS [1] and CMS [2] collaborations. This particle was observed in searches focused on di-bosonic decay modes of the Higgs boson, primarily $H \rightarrow \gamma \gamma, H \rightarrow Z Z^{(*)}$, and $H \rightarrow W W$, while no significant excesses were found yet in searches targeting fermionic decay modes $(H \rightarrow b \bar{b}$ and $\left.H \rightarrow \tau^{+} \tau^{-}\right)$. Therefore, it is imperative to continue to pursue searches in other production and decay modes over a wide mass range trying to establish if a signal is also present, and if so, whether the estimated mass is consistent with $m_{H} \approx 125 \mathrm{GeV}$.

A search for $t \bar{t} H(H \rightarrow b \bar{b})$ production in $p p$ collisions at $\sqrt{s}=7 \mathrm{TeV}$ using data corresponding to an integrated luminosity of $4.7 \mathrm{fb}^{-1}$ collected by the ATLAS [3] experiment is presented [4]. This search is simultaneously sensitive to the Yukawa coupling between the top quark and the Higgs boson and the $H \rightarrow b \bar{b}$ branching ratio. The only assumption made is that the Higgs boson is a narrow scalar particle.

\section{Analysis Overview}

The analysis is focused on the semileptonic decay mode of the $t \bar{t}$ system, where the $W$ boson from one top quark decays to a charged lepton and its associated neutrino, and the $W$ boson from the other top quark decays to a quarkantiquark pair. The events are identified by a high transverse momentum $\left(p_{\mathrm{T}}\right)$ electron or muon, a characteristic

\footnotetext{
a e-mail: javier.montejo.berlingen@ @ern.ch
}

that is crucial to triggering, high missing transverse momentum $\left(E_{\mathrm{T}}^{\text {miss }}\right)$ from the undetected neutrino, and a high jet multiplicity. They are also distinguished by a large number of $b$-quark-initiated jets, which helps to further distinguish them from the backgrounds. The main background to the search comes from $t \bar{t}$ events where there are at least two extra jets produced in association with the top quarks. The amount of this background depends on the $b$-jet identification efficiency and misidentification rate of light jets provided by the $b$-tagging algorithm used, and the number of reconstructed $b$ jets required in the analysis. A fraction of the $t \bar{t}+$ jets background consists of $t \bar{t} b \bar{b}$, which constitutes an irreducible background.

Events with one high- $p_{\mathrm{T}}$ isolated electron or muon, high $E_{\mathrm{T}}^{\mathrm{miss}}$ and at least four reconstructed jets are selected, and they are categorised into nine different topologies depending on their jet and $b$-tagged jet multiplicities. Two different discriminants are employed depending on the category. Categories with fewer than six jets or fewer than three $b$-tagged jets are dominated by background and the discriminant used is $H_{\mathrm{T}}^{\text {had }}$, the scalar sum of the jet transverse momenta, $p_{\mathrm{T}}^{\text {jet }}$. $H_{\mathrm{T}}^{\text {had }}$ is primarily sensitive to uncertainties associated with jet reconstruction and measurement, as well as with the modelling of the $t \bar{t}+$ jets background. Categories with at least six reconstructed jets and with three or more $b$-tagged jets present the highest signalto-background ratio and have the highest sensitivity to a Higgs boson signal. In these categories a kinematic fit of the event is performed in order to identify the Higgs decay. 


\section{Background and Signal Modeling}

The main physics background to this search is $t \bar{t}+\mathrm{jets}$ production. Smaller background contributions arise from $W+$ jets, single top, diboson ( WW, WZ, ZZ) and $Z+$ jets production. These physics backgrounds are predicted from the Monte Carlo (MC) simulation and are normalised to higher-order theoretical cross sections. In the case of $W+$ jets, the shapes of kinematic distributions are predicted with the simulation but the overall yield and flavour composition are determined using datadriven techniques based on the charge asymmetry between $W^{+}$and $W^{-}$productions. The multijet background cannot be modeled accurately with the MC simulation and is estimated using data-driven techniques.

Simulated samples of $t \bar{t} H \quad(H \rightarrow b \bar{b}) \quad$ signal are generated with the leading-order generator PythiA, for a range of Higgs boson masses: $m_{H}=$ $110,115,120,125,130,140 \mathrm{GeV}$. These samples are normalised to the next-to-leading order theoretical cross section. The cross sections and $H \rightarrow b \bar{b}$ branching ratio predictions used can be found in Ref. [5-10].

\subsection{Tag-Rate-Function Method}

When requiring high jet and $b$-tag multiplicity in the analysis, the available MC sample is significantly reduced, leading to large fluctuations in the resulting distributions. This can negatively affect the sensitivity of the analysis, as the corresponding statistical uncertainties on the background templates need to be taken into account in the determination of exclusion limits, and lead to unreliable systematic uncertainties in the predicted distribution shapes. In addition, the observed limits may be biased, depending on how the MC distributions fluctuate with respect to the data in the signal region.

In order to mitigate this problem, a tag-rate-function (TRF) method was developed to provide a $b$-tagging probability for each jet in the pre- $b$-tagged sample. This allows the use of the full MC sample in the prediction of the normalisation and shape of the signal and backgrounds after $b$-tagging.

By using the TRF method, no event is cut based on its b-tagging count, but instead all the events are weighted. This weight can be interpreted as the probability of the given event to contain the desired number of $b$ jets. To achieve this, the tagging efficiency (a function of $\eta, p_{\mathrm{T}}$ and true jet flavour) is used to calculate the event weight based on the kinematics and flavour of the jets found in each event.

\section{Kinematic Reconstruction}

A natural discriminator between the $t \bar{t} H(H \rightarrow b \bar{b})$ signal and the background is the reconstructed invariant mass of the $b$-quark pair, $m_{b \bar{b}}$, in particular if the $b$-quarks can be determined to not have originated from the top quarks. This assignment can be made for events with $\geq 6$ jets of which $\geq 3$ jets are $b$ tagged, from the two jets that are not assigned to the $t \bar{t}$ system. In the case of only three $b$ tagged jets one jet in either the top quark or Higgs boson system will not be $b$ - tagged. In these cases a kinematic fit via a maximum likelihood approach [11] is performed to select the best assignment of four of the six jets to the two top quarks in the final state. Their energies and transverse momenta are adjusted to reconstruct the correct masses for the various resonances present in the $t \bar{t}$ hypothesis, according to the mass width and experimental resolution. The invariant mass of the two jets not included in the $t \bar{t}$ reconstruction $\left(m_{b \bar{b}}\right)$ is analysed in the search for a Higgs boson resonance.

\section{Systematic Uncertainties}

Several sources of systematic uncertainties have been considered that can affect the normalisation of signal and background and/or the shape of their corresponding final discriminant distributions. Individual sources of systematic uncertainty are considered uncorrelated. Correlations of a given systematic uncertainty are maintained across processes and channels. The dominating systematics are $t \bar{t}+$ heavy-flavour modeling, $b-, c$-, and light-tagging efficiencies, multijet normalisation, the $t \bar{t}+$ light flavour modelling and jet energy scale. In order to reduce the degrading impact of systematic uncertainties on the sensitivity of the search, nuisance parameters corresponding to each of the systematic uncertainties are fitted to data during the statistical analysis, exploiting the constraining power from the background-dominated topologies considered.

\section{Results}

A simultaneous fit to the background-dominated topologies and those with signal is performed to obtain an improved background prediction with reduced uncertainties, resulting in a better search sensitivity. Hypothesis testing is performed using a modified frequentist method as implemented in RooStats [12] and based on a profile likelihood which takes into account the systematic uncertainties as nuisance parameters which are fitted to the data. This procedure allows the degrading impact of systematic uncertainties on the search sensitivity to be minimized, by taking advantage of the high-statistics backgrounddominated control channels included in the likelihood fit. The distributions of the final discriminants from each of the channels considered are combined in the statistical analysis to test for the presence of a signal at each value of the hypothesized Higgs boson mass.

Observed and expected 95\% confidence level (CL) upper limits on the Higgs boson production cross section times its branching ratio for decays to $b$-quark pairs, $\sigma(t \bar{t} H) \times B R(H \rightarrow b \bar{b})$. These are shown in Table 1 as a ratio of the derived limits to the SM prediction, as a function of $m_{H}$.

\section{Conclusions}

A search has been performed for a Higgs boson produced in association with a pair of top quarks $(t \bar{t} H)$ and decay- 
ing into a pair of bottom quarks $(H \rightarrow b \bar{b})$, using $4.7 \mathrm{fb}^{-1}$ of $p p$ collisions at $\sqrt{s}=7 \mathrm{TeV}$ collected in 2011 with the ATLAS detector at the CERN Large Hadron Collider. The search is focused on the semileptonic decay mode of the $t \bar{t}$ system and combines nine different topologies given by the jet and $b$-tagged jet multiplicities of the event. For events with at least six jets and three or more $b$-tagged jets, a kinematic reconstruction of the Higgs boson candidate is performed, whose invariant mass becomes the primary discriminant variable between signal and background. Background-dominated samples with lower jet and/or $b$-tagged jet multiplicities are exploited to constrain in-situ the leading systematic uncertainties affecting the background prediction. No significant excess of events above the background expectation is observed and $95 \%$ confidence-level upper limits on $\sigma(t \bar{t} H) \times B R(H \rightarrow b \bar{b})$, are derived for a Higgs boson with a mass between 110 and $140 \mathrm{GeV}$. For $m_{H}=125 \mathrm{GeV}$, an observed (expected) $95 \%$ confidence-level upper limit of 13.1 (10.5) times the SM Higgs boson cross section is obtained.

\begin{tabular}{|c|c|c|c|}
\hline \hline$m_{H}(\mathrm{GeV})$ & observed & expected & stat only \\
\hline 110 & 7.0 & 6.0 & 3.5 \\
115 & 8.7 & 6.9 & 4.0 \\
120 & 10.4 & 8.5 & 4.9 \\
125 & 13.1 & 10.5 & 6.1 \\
130 & 16.4 & 13.0 & 7.8 \\
140 & 33.0 & 23.2 & 14.2 \\
\hline
\end{tabular}

Table 1. Observed and expected 95\% CL upper limits on $\sigma(t \bar{t} H) \times B R(H \rightarrow b \bar{b})$ relative to the SM prediction, $\sigma / \sigma_{S M}$, as functions of $m_{H}$. The last column corresponds to the expected limit with all systematic uncertainties removed.

\section{References}

[1] ATLAS (ATLAS collaboration), Phys. Lett. B716, 1 (2012)

[2] CMS (CMS collaboration), Phys. Lett. B716, 30 (2012)

[3] ATLAS (ATLAS collaboration), S08003, 3 (2008)

[4] ATLAS-COM-CONF-2012-162 https: //cdsweb.cern.ch/record/1478423

[5] S. Dittmaier et al. (LHC Higgs Cross Section Working Group) (2011)

[6] Z. Kunszt, Nucl. Phys. B 247, 339 (1984)

[7] W. Beenakker et al., Phys. Rev. Lett. 87, 201805 (2001)

[8] W. Beenakker et al., Nucl. Phys. B 653, 151 (2003)

[9] S. Dawson, L. H. Orr, L. Reina, and D. Wackeroth, Phys. Rev. D 67, 071503 (2003)

[10] S. Dawson, C. Jackson, L. Orr, L. Reina, and D. Wackeroth, Phys.Rev. D 68, 034022 (2003)

[11] ATLAS-CONF-2011-033 (2011)

[12] W. Verkerke, D. Kirkby (RooFit Users Manual v2.91) (2012) 